\title{
Design and Construction of the Mechanical Structure of an SCARA Type Arm for Didactic Use
}

\author{
Miguel Magos Rivera, Doctorado ${ }^{1}$, José Antonio Lara Chávez, Licenciatura ${ }^{1}$, Salvador Piñones Contreras, \\ Licenciatura $^{1}$, and Ricardo Godínez Bravo, Licenciatura ${ }^{1}$ \\ ${ }^{1}$ Universidad Autónoma Metropolitana - Azcapotzalco, Ciudad de México, México \\ mrm@azc.uam.mx, jalch@azc.uam.mx, rgb@azc.uam.mx
}

\begin{abstract}
Robotics is one of the branches of engineering that will play a very important role in the Industry 4.0 scheme. One way for Higher Education Institutions to have robotic devices without incurring high costs is through self-equipment. This paper presents the design and construction of the mechanical structure of a robotic arm type SCARA for teaching applications. The prototype has 3 degrees of freedom and a maximum operating radius of $35 \mathrm{~cm}$. Using a suction cup, it is possible to move objects with a maximum weight of 200 grams. Arm movements are performed by stepper motors. Each degree of freedom has associated limit switches that will serve to limit the movements of the robotic arm when controlling its operation. Preliminary tests of operation show that the movements of the system are correct and are within the established values for the equipment.
\end{abstract}

Keywords- SCARA Robot, Computer aided design, Mechatronic, Teaching prototype.

Digital Object Identifier (DOI):

http://dx.doi.org/10.18687/LACCEI2020.1.1.600

ISBN: 978-958-52071-4-1 ISSN: 2414-6390

18th LACCEI International Multi-Conference for Engineering, Education, and Technology: "Engineering, Integration, and Alliances for a Sustainable Development" "Hemispheric Cooperation for Competitiveness and Prosperity on a Knowledge-Based Economy", July 27-31, 2020, Virtual Edition. 


\title{
Diseño y Construcción de la Estructura Mecánica de un Brazo Tipo SCARA para Uso Didáctico
}

\author{
Miguel Magos Rivera, Doctorado ${ }^{1}$, José Antonio Lara Chávez, Licenciatura ${ }^{1}$, Salvador Piñones Contreras, \\ Licenciatura ${ }^{1}$, and Ricardo Godínez Bravo, Licenciatura ${ }^{1}$ \\ ${ }^{1}$ Universidad Autónoma Metropolitana - Azcapotzalco, Ciudad de México, México \\ mrm@azc.uam.mx, jalch@azc.uam.mx,rgb@azc.uam.mx
}

\begin{abstract}
Resumen-La robótica es una de las ramas de la ingeniería que jugará un papel muy importante en el esquema de Industria 4.0. Una forma para que Instituciones de Educación Superior cuenten con equipos robóticos sin incurrir en elevados gastos es mediante el auto-equipamiento. Este artículo presenta el diseño y la construcción de la estructura mecánica de un brazo robótico tipo SCARA para aplicaciones didácticas. El prototipo tiene 3 grados de libertad y un radio de desplazamiento máximo de 35 cm. Usando una ventosa de vacío, es posible mover objetos de hasta 200 gr. Los movimientos del brazo se realizan por medio de motores a pasos. Cada grado de libertad tiene asociado sensores de límite que servirán para acotar los movimientos del brazo una vez que se realice el control de su operación. Pruebas preliminares de funcionamiento mostraron que los movimientos del sistema son correctos y se encuentran dentro de los valores establecidos para el equipo.
\end{abstract}

Keywords --Brazo SCARA, Diseño asistido por computadora, Mecatrónica, Prototipo didáctico.

\section{INTRODUCCIÓN}

Con el avance del concepto de industria 4.0, una de las áreas que mayor crecimiento presentará en los próximos años será la relacionada con la robótica industrial. Al integrar a estos equipos los avances tecnológicos actuales tales como: reconocimiento de imágenes, sensores inteligentes, deep learning, big data, etc., los robots serán cada vez más versátiles y fácil de manejar [1]. Al contar con los datos generados por los sensores involucrados en su operación, los robots serán más fáciles de configurarse por medio de auto aprendizaje en lugar de una programación previa. La integración de funciones de comunicación en todos los elementos relacionados con la operación de una planta industrial, está haciendo que el concepto de robótica colaborativa sea cada vez más común [2]. Robots trabajando entre sí en forma coordinada e inteligente, así como con el personal se reflejará en la optimización de la producción [3]. Todo lo anterior desembocará a futuro en plantas reconfigurables en donde la fabricación de productos bajo demanda sea una realidad.

Según datos de la International Federation of Robotics, en el periodo 2012 - 2017, la venta de robots a nivel mundial tuvo un incremento anual del 19\%. La misma fuente prevé un crecimiento anual promedio del $14 \%$ en el periodo 2018 - 2021 [4]. El panorama para la robótica industrial es, como puede verse, muy prometedor.

Digital Object Identifier (DOI)

http://dx.doi.org/10.18687/LACCEI2020.1.1.600

ISBN: 978-958-52071-4-1 ISSN: 2414-6390
La situación descrita traerá como consecuencia que a futuro los requerimientos de personal capacitado en el diseño, construcción, manejo y programación de robots de tipo industrial ira en aumento. Para los centros de capacitación, así como para las instituciones de educación superior, contar con equipos robóticos dedicados a la enseñanza implica un costo muy elevado. Una solución parcial a este problema se puede encontrar en el auto equipamiento. En el Departamento de Electrónica de la Universidad Autónoma Metropolitana campus Azcapotzalco en la Ciudad de México, se propuso el diseño y la construcción de un brazo robótico tipo SCARA que sirva de apoyo en cursos relacionados con Robótica Industrial, Automatización Industrial y Mecatrónica, entre otros. En este trabajo se presenta el diseño y construcción de la parte mecánica de este dispositivo.

Son diversos los equipos de investigación en el mundo que han trabajado en el diseño y la construcción de brazos robóticos tipo SCARA para aplicaciones didácticas. En la literatura es posible encontrar prototipos de 3 grados de libertad [5], [6]. Versiones con 4 grados también han sido desarrolladas, en [7] y [8] se describen dos ejemplos. Con la finalidad de poder realizar estudios sobre sistemas con mayor grado de complejidad, algunos grupos de investigadores han desarrollado brazos SCARA de 5 y 6 grados de libertad, algunos ejemplos se describen en [9], [10], [11], [12].

\section{DESCRIPCIÓN GENERAL}

El brazo tipo SCARA que se describe en este artículo, está diseñado para aplicaciones de sujetar y desplazar objetos (Pick and Place) mediante una ventosa conectada a una bomba de vacío. El mecanismo cuenta con 3 grados de libertad (GDL) en una configuración de dos articulaciones rotatorias paralelas (punto 1 y 2 ) y una articulación prismática (punto 3), mismas que se muestran en la Fig. 1.

El brazo robótico tiene un alcance horizontal de $35 \mathrm{~cm}$ medido entre el eje de soporte principal y el de la herramienta de sujeción. Verticalmente la ventosa tiene un rango de desplazamiento de $20 \mathrm{~cm}$ y puede soportar objetos que cuenten, por lo menos, con un área horizontal lisa de $45 \mathrm{~mm}$ de diámetro y con peso máximo de $200 \mathrm{gr}$, Fig. 2.

El rango de desplazamiento angular del brazo es de $160^{\circ}$, mientras que el del antebrazo es de $220^{\circ}$, lo anterior puede observarse en la vista superior del dispositivo que se muestra en la Fig. 3. 


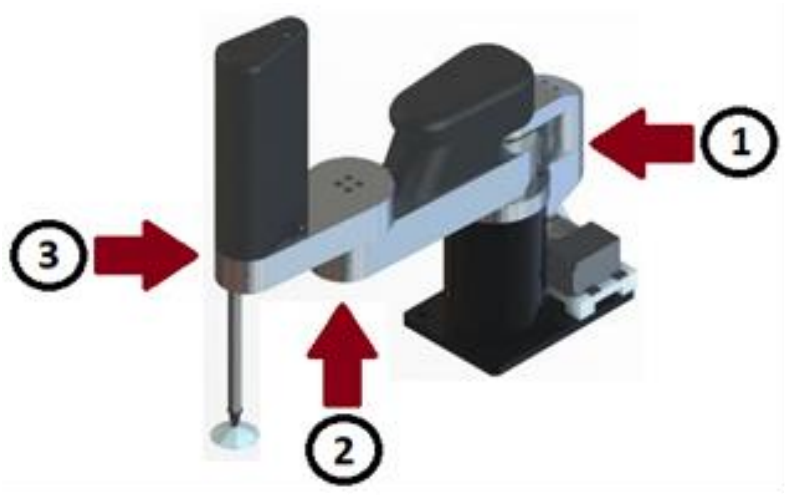

Fig. 1 Vista del Brazo SCARA construido.

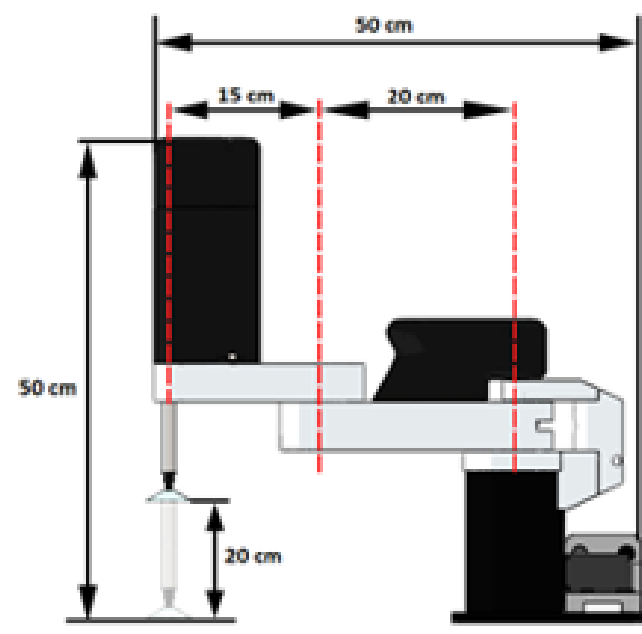

Fig. 2 Rangos de operación lineal del brazo SCARA.

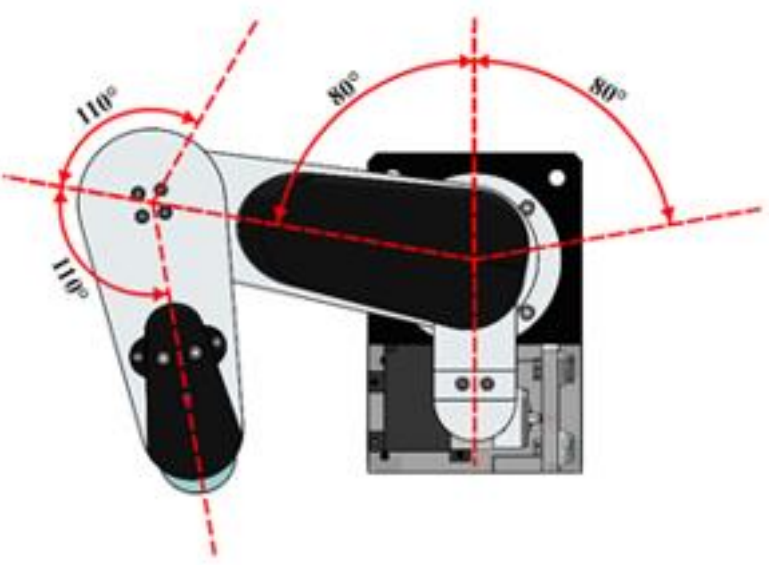

Fig. 3 Rangos de operación angular del brazo SCARA.

Por su parte, la Fig. 4 muestra el espacio de trabajo del prototipo construido. Como en la mayoría de los brazos SCARA, se trata de un segmento de cilindro hueco cuyo radio exterior depende del largo del brazo y del antebrazo. Mientras que el radio interior de dicho cilindro está asociado únicamente al largo del antebrazo. La altura del segmento de cilindro depende directamente del desplazamiento vertical de la ventosa del brazo robótico. Para asegurar que el brazo se desplace dentro de los rangos definidos y así evitar daño mecánico, el equipo cuenta con 3 pares de sensores de límite, uno para cada grado de libertad.

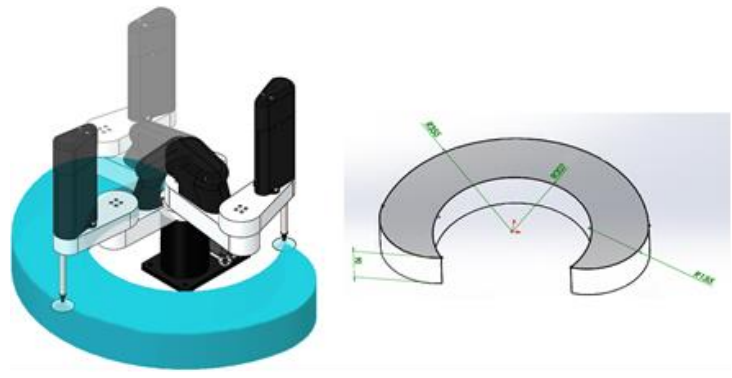

Fig. 4 Espacio de trabajo del brazo SCARA.

Para una mejor explicación de la construcción de las partes que conforman el equipo desarrollado, éste se dividió en cuatro grandes bloques: base, brazo, antebrazo y conectores. Estos subensambles se muestran en la Fig. 5 y se describen en las siguientes secciones de este documento.

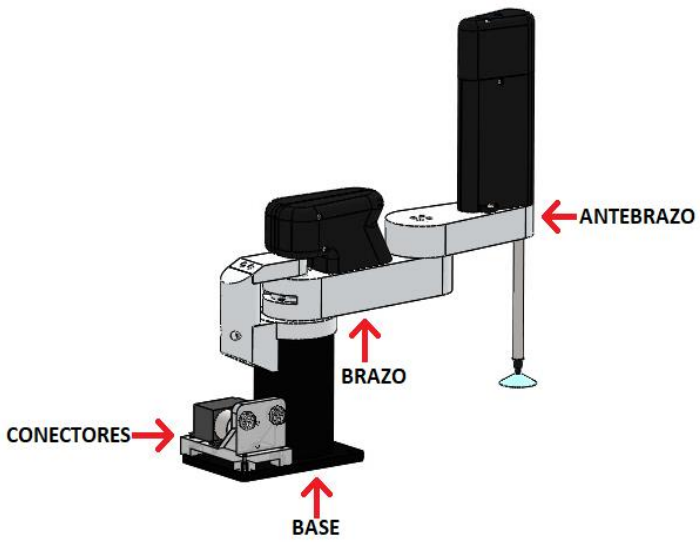

Fig. 5 Sub-ensambles del brazo robótico SCARA.

\section{Base}

Se trata del soporte del mecanismo completo, está fabricado principalmente en acero con la finalidad de que cuente con un peso considerablemente superior al resto del equipo y de esta forma se incremente su estabilidad. Este subensamble tiene perforaciones en su placa base para poder fijarlo a una superficie o mesa de trabajo, Fig. 6.

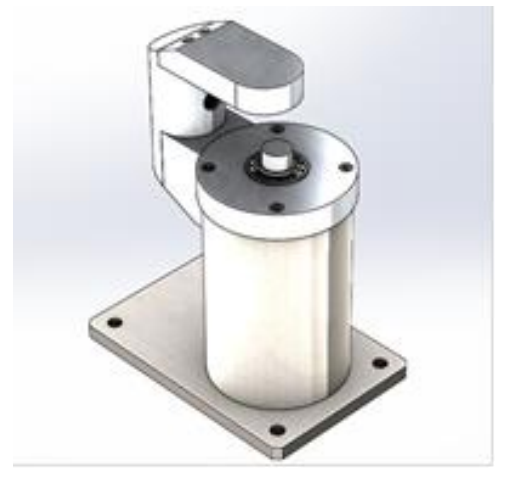

Fig. 6 Base del brazo robótico SCARA.

El subensamble está conformado por diversos elementos mecánicos los cuales se listan y observan en la vista explosionada de la Fig. 7. 


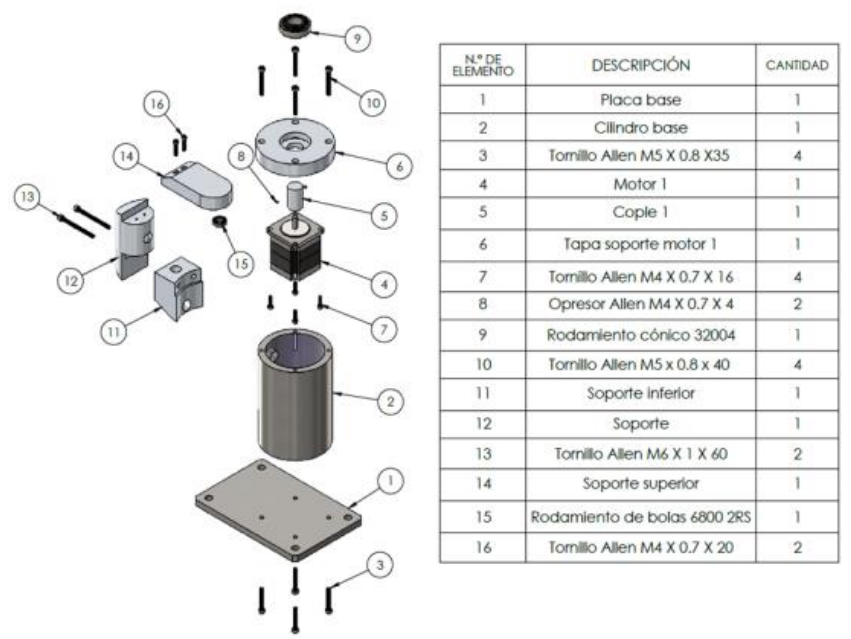

Fig. 7 Elementos del sub-ensamble Base del brazo robótico SCARA.

En este subensamble se encuentra alojado el motor que se encarga de transmitir el movimiento rotatorio al siguiente subensamble: el Brazo. Lo anterior se logra uniendo directamente el eje del motor al eje de giro del Brazo, esto vía un rodamiento cónico. Se utilizó un motor a pasos bipolar de 200 pasos por vuelta, su consumo de corriente es de $2 \mathrm{~A}$, y puede proporcionar un torque de $0.9 \mathrm{~N} / \mathrm{m}$. Así mismo, como se mencionó anteriormente, para evitar daño mecánico y tener un arco de giro definido, el subensamble cuenta con sensores de límite. La ubicación de estos se muestra en la Fig. 8.

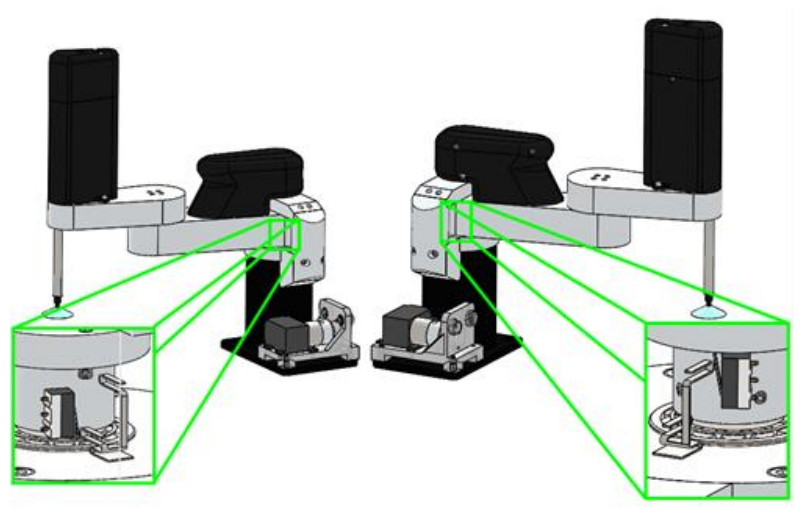

Fig. 8 Sensores de límite en la base del brazo robótico SCARA.

De igual forma en la base se encuentra la bomba de vacío, que en conjunto con la ventosa, se encarga de sujetar el objeto a trasladar. El dispositivo que se utilizó fue el modelo KYK50BPM de la marca Keyukang, la cual se alimenta con 12 VDC @ $850 \mathrm{~mA}$ y puede generar una presión máxima de vacío de $66 \mathrm{kPa}$ con un flujo de aire de 12 L/min. La ubicación de esta puede observarse en la Fig. 9.

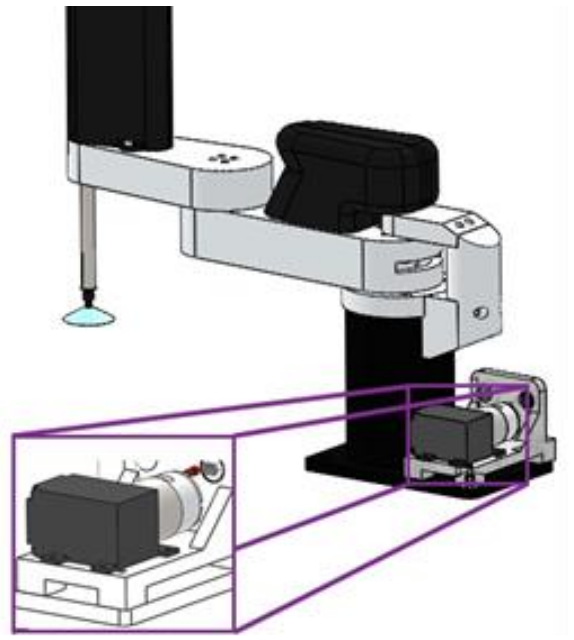

Fig. 9 Ubicación de la bomba de vacío.

\section{Brazo}

El brazo es el eslabón móvil que soporta al antebrazo, incluido su sistema de tracción, en la Fig. 10 se observa este subsistema. En la parte inferior se tiene la estructura principal, fabricada en aluminio, que se encarga de soportar a los elementos que conforman este bloque. En la parte superior se encuentra una tapa que cubre al motor a pasos que se encuentra integrado, en este caso la cubierta fue elaborada mediante impresión $3 \mathrm{D}$, por lo que se trata de material plástico.

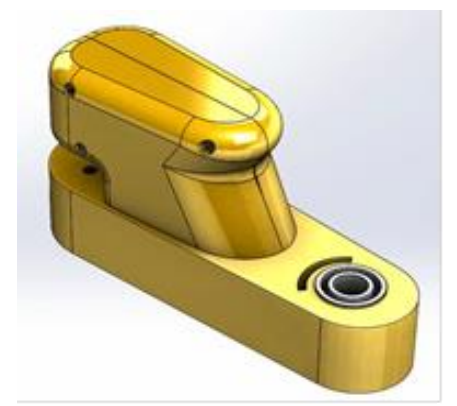

Fig. 10 Brazo del SCARA.

Los elementos que componen al Brazo se listan y observan en la vista explosionada de la Fig. 11.

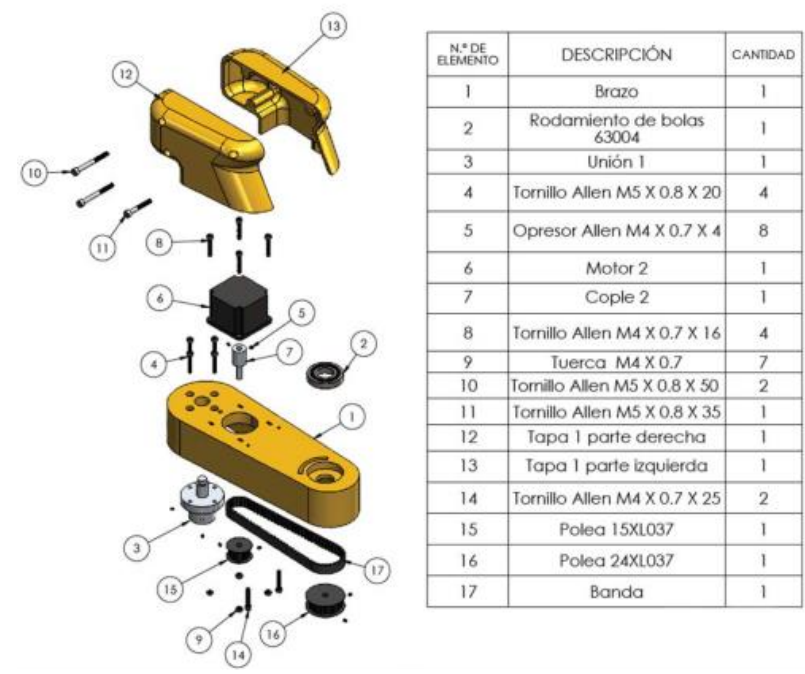

Fig. 11 Elementos del brazo del SCARA.

$1^{\text {th }}$ LACCEI International Multi-Conference for Engineering, Education, and Technology: "Engineering, Integration, and Alliances for a Sustainable Development" "Hemispheric Cooperation for Competitiveness and Prosperity on a Knowledge-Based Economy", 29-31 July 2020, Buenos Aires, 
El motor utilizado en esta parte del prototipo se encarga de transmitir el movimiento al siguiente eslabón del equipo: el Antebrazo. Lo anterior se logra mediante un par de poleas unidas por una banda dentada. Aquí también se empleó un motor a pasos bipolar de 200 pasos por vuelta, con consumo de corriente de $2 \mathrm{~A}$, pero en este caso el torque es menor $0.71 \mathrm{~N} / \mathrm{m}$. La ubicación del motor a pasos descrito puede observarse en la Fig. 12.

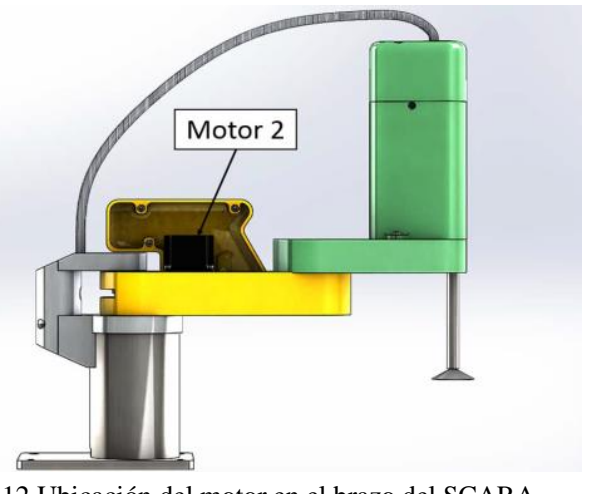

Fig. 12 Ubicación del motor en el brazo del SCARA.

Los sensores de límite que restringen el ángulo de trabajo se muestran en la Fig. 13.

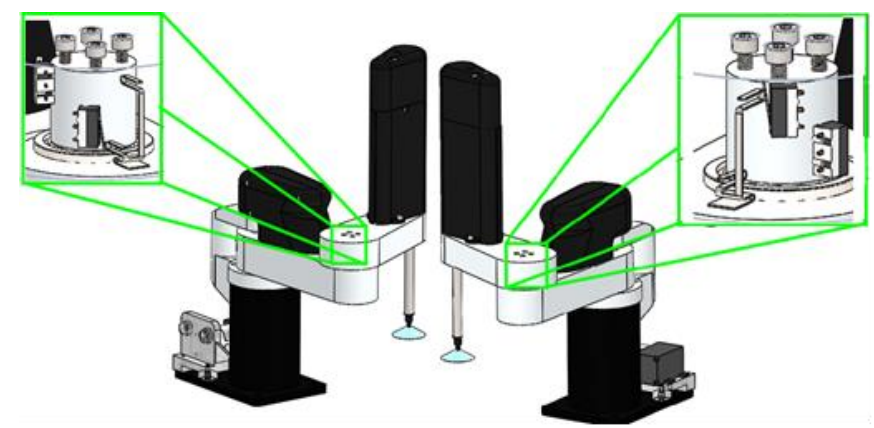

Fig. 13 Sensores de límite en el brazo del SCARA.

\section{Antebrazo}

El Antebrazo es el encargado de soportar al sistema de sujeción de objetos. En la Fig. 14 se observa este subensamble, en la parte inferior se tiene el soporte principal fabricado en aluminio, mientras que en la parte superior se observa la cubierta del mecanismo que proporciona movimiento al último grado de libertad. Mediante un tercer motor a pasos un eje es desplazado verticalmente llevando en su extremo inferior la ventosa que sujetará al objeto a transportar.

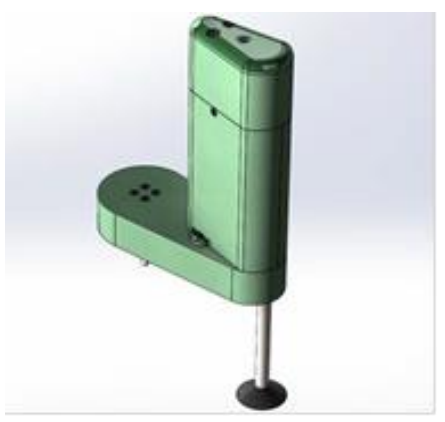

Fig. 14 Antebrazo del SCARA.
Los elementos que componen al Antebrazo se listan y observan en la vista explosionada de la Fig. 15.

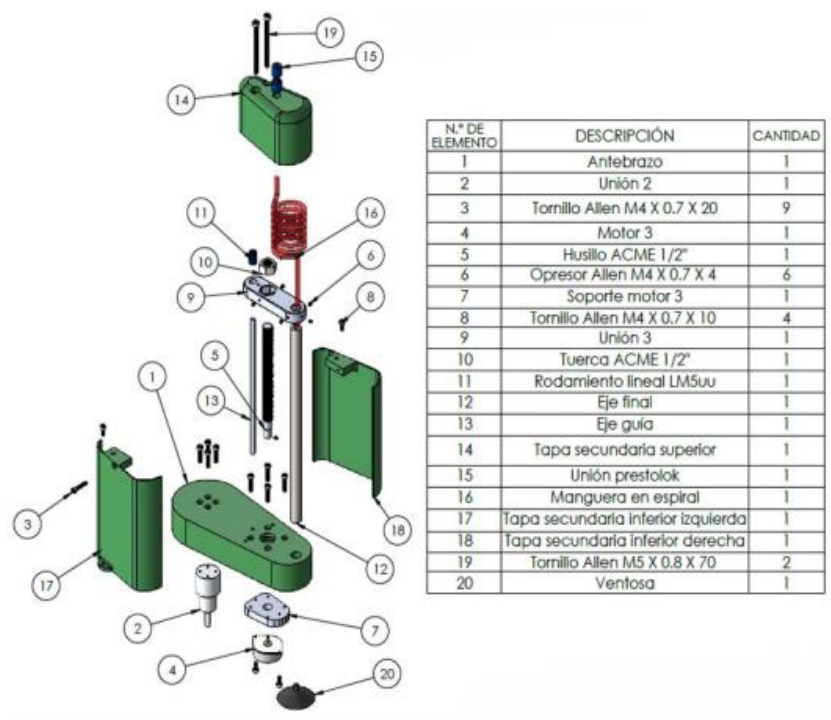

Fig. 15 Elementos del antebrazo del SCARA.

El motor utilizado para generar el movimiento lineal del eje que soporta en su extremo inferior a la ventosa, es también a pasos, en este caso se trata de un motor de 48 pasos por vuelta con un consumo de corriente de $0.4 \mathrm{~A}$., y un torque de $0.1 \mathrm{~N} / \mathrm{cm}$. Su ubicación se muestra en la Fig. 16. En esta misma figura se observa la manguera de plástico que conecta a la ventosa con la bomba de vacío, ubicada en la base del prototipo.

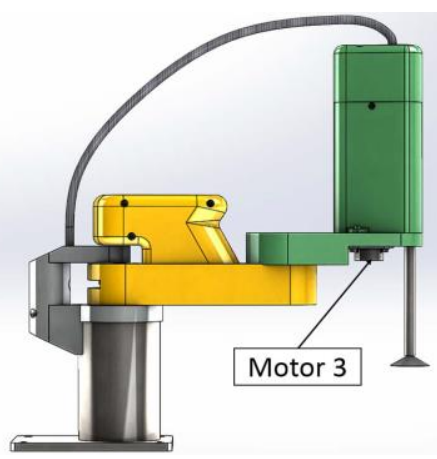

Fig. 16 Ubicación del motor en el brazo del SCARA.

En la Fig. 17, se muestran los sensores de límite que acotan el desplazamiento vertical del eje que soporta a la ventosa.

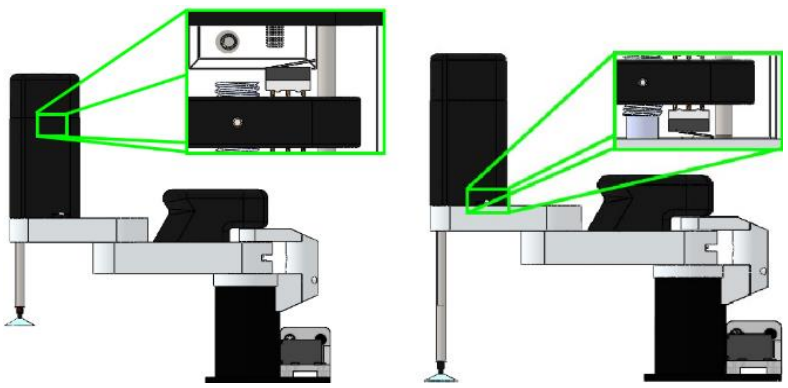

Fig. 17 Sensores de límite en el antebrazo del SCARA.

$1^{\text {th }}$ LACCEI International Multi-Conference for Engineering, Education, and Technology: "Engineering, Integration, and Alliances for a Sustainable Development" "Hemispheric Cooperation for Competitiveness and Prosperity on a Knowledge-Based Economy", 29-31 July 2020, Buenos Aires, 


\section{Conectores}

Con la finalidad de que un dispositivo externo pueda controlar el prototipo, se tienen dos conectores hembra redondos de aviación modelo GX en una base de acrílico fija en la base del prototipo. En el primero de ellos, de 10 terminales, se reciben las señales provenientes de los sensores de límite. Mientras que en el segundo, de 15 terminales, se tienen disponibles las terminales de los distintos motores del equipo: bomba de vacío y motores a pasos. La Fig. 18 muestra la ubicación de dichos conectores, mientras que en las tablas 1 y 2 se especifica la distribución de las terminales en los conectores.

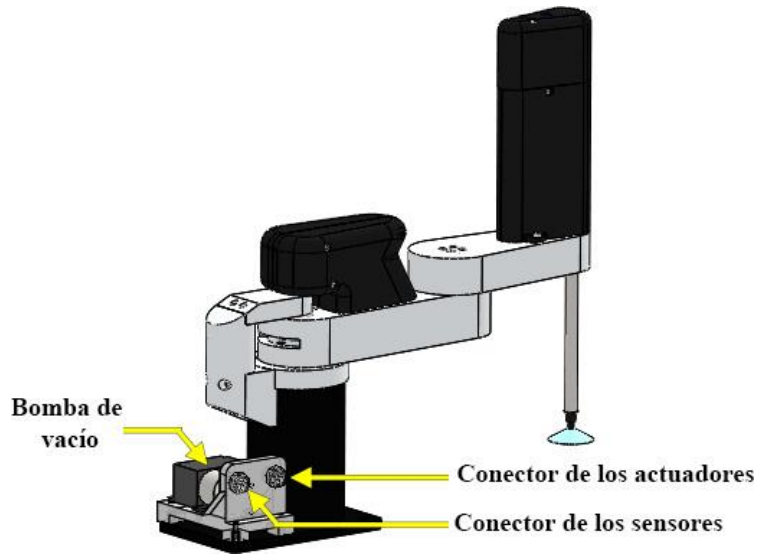

Fig. 18 Ubicación de los conectores en el brazo SCARA.

TABLA I

DisTRIBUCIÓN DE PINES CONECTOR GX-10

\begin{tabular}{|c|l|}
\hline PIN & \multicolumn{1}{|c|}{ Descripción } \\
\hline 1 & Sensor límite izquierdo de la base \\
\hline 2 & Sensor límite derecho de la base \\
\hline 3 & Sensor límite izquierdo del brazo \\
\hline 4 & Tierra \\
\hline 5 & No utilizado \\
\hline 6 & No utilizado \\
\hline 7 & No utilizado \\
\hline 8 & Sensor límite derecho del brazo \\
\hline 9 & Sensor límite superior del antebrazo \\
\hline 10 & Sensor límite inferior del antebrazo \\
\hline
\end{tabular}

TABLA II

\begin{tabular}{l} 
DISTRIBUCIÓN DE PINES CONECTOR GX- \\
\begin{tabular}{|c|l|}
\hline PIN & Descripción \\
\hline 1 & Bomba de vacío (V+) \\
\hline 2 & Motor Base (A-) \\
\hline 3 & Motor Brazo (A-) \\
\hline 4 & Motor Antebrazo (A-) \\
\hline 5 & Motor Antebrazo (A+) \\
\hline 6 & No utilizado \\
\hline 7 & Motor Antebrazo (B+) \\
\hline 8 & Motor Brazo (B+) \\
\hline 9 & Motor Base (B+) \\
\hline 10 & Bomba de vacío (V-) \\
\hline 11 & Motor Base (A+) \\
\hline 12 & Motor Brazo (A+) \\
\hline 13 & Motor Antebrazo (B-) \\
\hline 14 & Motor Brazo (B-) \\
\hline 15 & Motor Base (B-) \\
\hline
\end{tabular} \\
\hline
\end{tabular}

\section{Resultados}

El prototipo, una vez diseñado mediante el paquete de cómputo SolidWorks, fue construido empleando diversos materiales, tal y como fue descrito en las distintas secciones de este artículo. Principalmente se utilizó aluminio, solo en la base del equipo se prefirió emplear acero para darle peso y estabilidad. Varias piezas fueron elaboradas mediante impresión 3D, estas básicamente fueron para cubrir algunos de los mecanismos del prototipo. Las especificaciones de movimiento planteadas inicialmente se cumplieron:

- Alcance horizontal de $35 \mathrm{~cm}$ medido entre el eje de soporte principal y el de la herramienta de sujeción.

- Desplazamiento vertical de la ventosa de $20 \mathrm{~cm}$.

- Desplazamiento angular del brazo de $160^{\circ}$.

- Desplazamiento angular del antebrazo de $220^{\circ}$.

El resultado obtenido fue el brazo robótico de tres grados de libertad que se muestra en la Fig. 19.

En esta parte del trabajo se desarrolló únicamente la estructura mecánica del brazo robótico, por lo que el equipo aún no cuenta con los sistemas de potencia y control para los actuadores. Se realizaron pruebas sencillas de funcionamiento para cada uno de los grados de libertad del prototipo, así como para el sistema de sujeción de este. En el caso de los movimientos angulares se observó la necesidad de algún tipo de freno, ya sea mecánico o eléctrico, que mantenga fijo al Brazo cuando el Antebrazo se desplace y viceversa. El problema anterior se plantea solucionarlo mediante el control de la corriente en las bobinas de los motores a pasos. El sistema de desplazamiento vertical tuvo un funcionamiento correcto en las pruebas realizadas. Por su parte en el sistema de vacío se constató que la sujeción de los objetos mejora en la medida que la superficie de contacto con la ventosa esté pulida. En pruebas preliminares se lograron desplazar objetos de hasta $350 \mathrm{~g}$. que cumplían con esta condición. En la Fig. 20 se muestra una vista de la bomba de vacío empleada colocada en la placa base del prototipo.

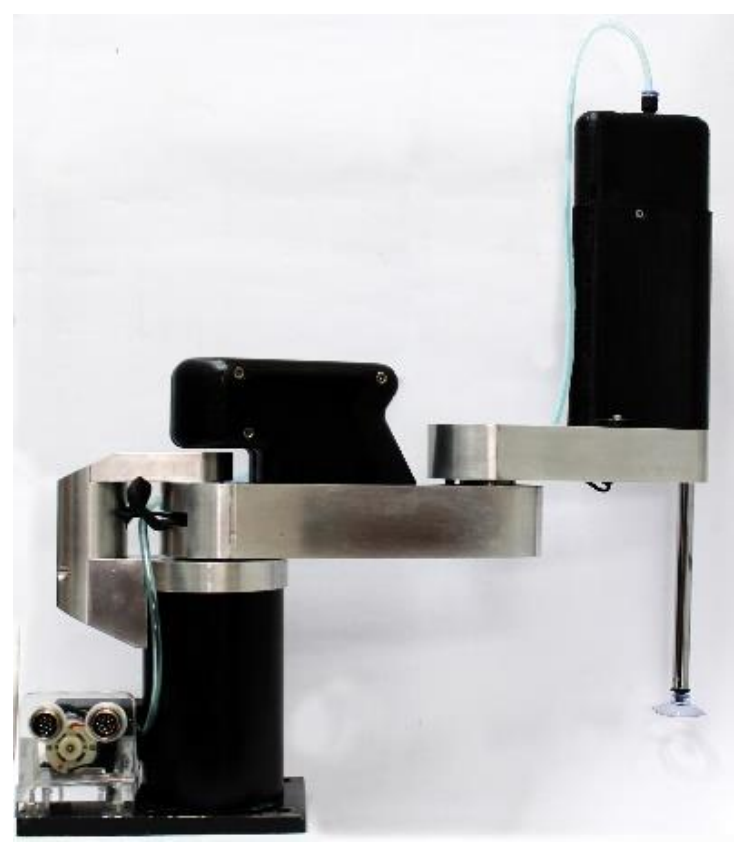

Fig. 19 Vista del robot tipo SCARA construido.

$1^{\text {th }}$ LACCEI International Multi-Conference for Engineering, Education, and Technology: "Engineering, Integration, and Alliances for a Sustainable Development" "Hemispheric Cooperation for Competitiveness and Prosperity on a Knowledge-Based Economy", 29-31 July 2020, Buenos Aires, 


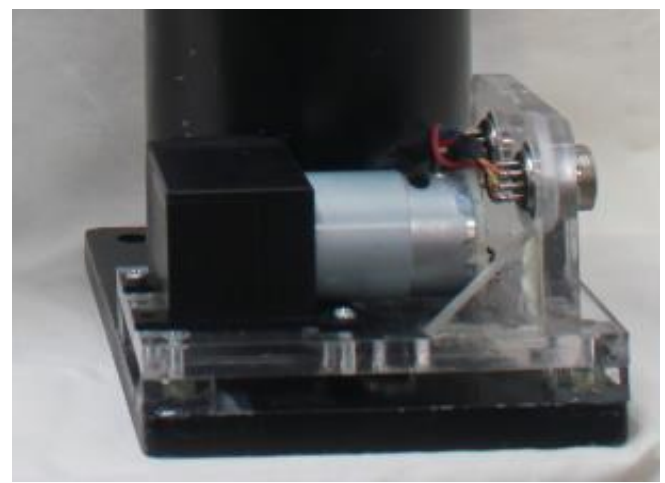

Fig. 20 Bomba de vacío

El llevar a un par de conectores las señales de los sensores y de los actuadores, facilitará a futuro la conexión de diversos tipos de controladores basados en diversas tecnologías. Una vista de los conectores instalados en el prototipo se observa en la Fig. 21.

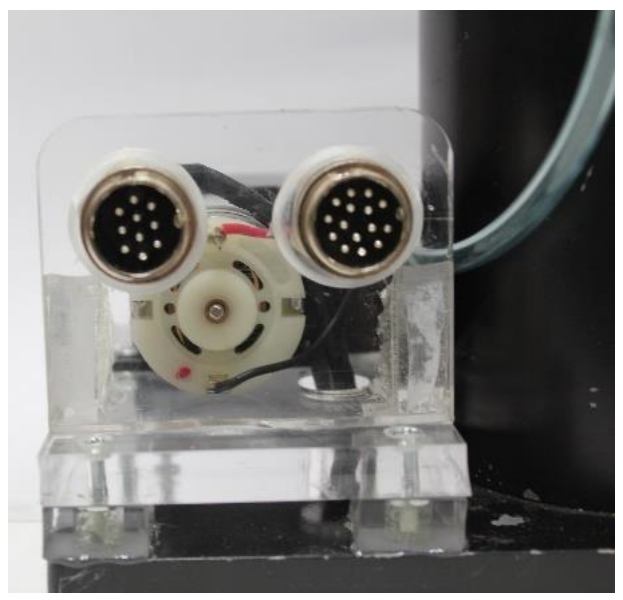

Fig. 21 Conectores de señales.

\section{Conclusiones}

En este trabajo se describió el diseño y construcción de la estructura mecánica de un brazo robótico tipo SCARA para aplicaciones didácticas. Desde el punto de vista mecánico, el equipo cumple con las especificaciones planteadas en los objetivos. Las pruebas preliminares que se realizaron a los subsistemas mostraron que los intervalos de movimiento son los deseados, además el mecanismo de sujeción de objetos trabaja adecuadamente, siempre y cuando exista una superficie plana para generar el vacío.

Al momento de redactar este artículo, se está iniciando el diseño de las etapas de potencia para los actuadores. En una primera instancia, se pretende emplear una computadora de una sola placa tipo Raspberry para realizar el control retroalimentado del brazo, quedando abierta la posibilidad de diseñar más adelante sistemas de control basados en Arduino, Microcontroladores, Computadora, etc.

Cabe mencionar que, el brazo robótico construido siempre tuvo como objetivo servir de apoyo para cursos en carreras de ingeniería relacionados con robótica industrial, control de procesos y automatización industrial, por mencionar algunos. Por lo anterior su capacidad, tanto de movimiento, como de carga es reducida lo que no lo hace apto para aplicaciones industriales. Sin embargo, el diseño realizado, mediante adecuaciones que incrementen su robustez y capacidad de carga, podría ser extrapolado para la construcción de equipos que pudiesen operar en aplicaciones de tipo industrial de bajo volumen.

\section{REFERENCIAS}

[1] K. Al-Gumaei, k. Schuba, S. Heymann, A. Friesen, C. Pieper, F. Pethig, and S. Schriegel, "A survey of Internet of Things and Big Data integrated solutions for industrie 4.0,"Procedia Engineering, vol. 182, pp. 763-769, December 2017.

[2] F. Zezulka, P. Marcon, Z. Bradac, J. Arm, T. Benesl, and I. Vesely, "Communication systems for industry 4.0 and the IIoT, IFAC PapersOnLine, vol. 51, no. 6, pp. 150-155, 2018.

[3] H. Du, W. Xu, B. Yao, Z. Zhou, and Y. Hu, "Collaborative optimization of service scheduling for industrial cloud robotics based on knowledge sharing," Procedia CIRP, vol. 83, pp. 132-138, 2019.

[4] International Federation of Robotics, Executive summary world robotics 2018, Frankfurt Main, Germany, October 2018.

[5] A. Gómez Espinosa, P. D. Lafuente Ramón, C. Rebollar Huerta, M. A. Hernández Maldonado, E. H. Olguín Callejas, H. Jiménez Hernández, E. A. Rivas Araiza, and J. Rodríguez Reséndiz, "Design and construction of a didactic 3-DOF parallel links robot station with a 1DOF gripper," Journal of Applied Research and Technology, vol. 12, no. 3, pp. 435-443, June 2014.

[6] A. Balaji, A. A. Nippun Kumaar, and T. S. B. Sudarshan, "3 axis Scara robot with universal gripper," International Conference on Trends in Automation, Communications and Computing Technology, Acharya, India, December 2015.

[7] M. Shariatee, A. Akbarzadeh, A. Mousavi, and S. Alimardani, "Design of an economical SCARA robot for industrial applications," 2nd International Conference on Robotics and Mechatronics, Teheran, Iran, October 2014

[8] L. Wen Bo, C. Guang Zhong, G. Xiao Qin, and H. Su Dan, "Development of a 4-DOF SCARA robot with 3R1P for pick-andplace tasks," 6th International Conference on Power Electronics Systems and Applications, Hong Kong, China, December 2015.

[9] M. Abu Qassem, I. Abuhadrous, and H. Elaydi, "Modeling and simulation of 5 DOF educational robot arm," 2nd International Conference on Advanced Computer Control, Shenyang, China, March 2010

[10] C. Feng, L. Jihao, Z. Chen, Z. Yanzheng, F. Zhuang, and Y. Weixin, "A novel 5-DOF welding robot based on SCARA," 10th Conference on Industrial Electronics and Applications, Auckland, New Zealand, June 2015

[11] M. B. D. S. Neto, J. M. C. D. Mendonça, and A. P. C. D. Sena, "Development and control of a prototype manipulator SCARA type as teaching tool," IFAC PapersOnLine, vol. 48, no. 19, pp. 209-213, 2015.

[12] C. Urrea, J. Cortés, and J. Pascal, "Design, construction and control of a SCARA manipulator with 6 degrees of freedom," Journal of Applied Research and Technology, vol. 14, no. 6, pp. 396-404, December 2016. 